Nouvelles perspectives en sciences sociales

\title{
La part mimétique des dynamiques de cognition sociale : clé pour penser l'auto-tranformation du social
}

\section{David Chavalarias}

Volume 2, numéro 2, mars 2007

URI : https://id.erudit.org/iderudit/602459ar

DOI : https://doi.org/10.7202/602459ar

Aller au sommaire du numéro

\section{Éditeur(s)}

Prise de parole

\section{ISSN}

1712-8307 (imprimé)

1918-7475 (numérique)

Découvrir la revue

\section{Citer cet article}

Chavalarias, D. (2007). La part mimétique des dynamiques de cognition sociale : clé pour penser l'auto-tranformation du social. Nouvelles perspectives en sciences sociales, 2(2), 33-57. https://doi.org/10.7202/602459ar
Résumé de l'article

Certains scientifiques, sociologues ou économistes en particulier, se sont récemment emparés du terme de cognition sociale utilisé en psychologie en lui donnant un second sens, à savoir, une cognition distribuée sur l'ensemble des individus composant une société. L’enjeu est alors de savoir « en quoi les aptitudes sociales humaines rendent compte du développement d'une cognition collective complexe ». Nous défendons ici l'idée que la cognition sociale est un raffinement du concept de cognition distribuée plutôt qu'un équivalent. C'est un phénomène propre aux sociétés humaines qui permet de penser leur propriété d'auto-transformation. Nous montrons comment l'imitation permet de penser ce phénomène, comme le suggérait en son temps Gabriel Tarde. 


\title{
La part mimétique des dynamiques de cognition sociale : clé pour penser l'auto-transformation du social
}

\author{
David Chavalarias ${ }^{1}$ \\ CREA, École polytechnique \\ Institut des systèmes complexes de Paris Île-de-France
}

Certains scientifiques, sociologues ou économistes se sont récemment emparés du terme de « cognition sociale » utilisé en psychologie ${ }^{2}$ en lui donnant un second sens, à savoir une cognition distribuée sur l'ensemble des individus composant une société. L'enjeu est alors de savoir « en quoi les aptitudes sociales humaines rendent compte du développement d'une

1 Cet article a bénéficié de l'influence de trop nombreuses personnes pour être mentionnées individuellement. Qu'elles en soient collectivement remerciées. Je remercic en particulicr l'ensemble des membres du CREA pour leurs discussions toujours stimulantes, Pascal Roggero pour son invitation dans le réseau AFS « sociologie et systèmes complexes » qui a suscité la rédaction de cet article, ainsi que les évaluateurs qui ont pris soin de relire l'article. Cet article a bénéficié du soutien de l'École Polytechnique et du CNRS.

2 Ensemble d'attitudes permettant la compréhension intuitive des relations sociales.

3 Paul Bourgine, "What is cognitive economics? ”, dans Paul Bourgine et Jean-Pierre Nadal (dir.), Cognitive economics. An interdisciplinary approach, Berlin, Springer, 2004, p. 1 12. 
cognition collective complexe ${ }^{4} »$. Nous défendons ici l'idée que la cognition sociale est un raffinement du concept de cognition distribuée plutôt qu'un équivalent. C'est un phénomène propre aux sociétés humaines. Cette notion doit, en effet, tenir compte d'un certain nombre de caractéristiques de la cognition humaine :

i. la reconnaissance de la part du sujet de l'autre comme acteur agissant dans et sur son environnement de manière intentionnelle,

ii. la prise en compte (par anticipation ou imitation) des croyances et des représentations des autres autant que de leurs actions,

iii. la modification de la constitution même du sujet (ses croyances, ses représentations, ses règles, ses buts, etc.) au cours de ses interactions sociales.

Nous tenterons ici d'approfondir le concept de cognition sociale ainsi que les apports potentiels d'une approche systèmes complexes en rendant hommage à l'un des précurseurs de la cognition sociale, trop vite oublié de son temps, Gabriel Tarde. La première section pose la cognition sociale comme un raffinement de la notion de cognition distribuée en insistant sur la propriété d'auto-transformation comme trait spécifique. La deuxième section montre en quoi l'imitation est à même de rendre compte de cette spécificité dans les dynamiques sociales qu'elle définit. Enfin, la troisième section présente un modèle multi-agents de ces dynamiques mimétiques susceptible d'illustrer cette propriété d'auto-transformation.

\section{Cognition distribuée et cognition sociale}

La cognition distribuée peut être définie comme un traitement de l'information effectué par un ensemble d'entités éventuellement hétérogène. Par exemple, Hutchins ${ }^{5}$ défend l'idée d'un traitement de l'information au sein d'un système hétérogène homme/machine. Lorsque que le système est homogène, on emploie souvent le terme de « cognition collective » ou celui d'« intelligence collective », comme c'est le cas pour l'étude des comportements collectifs des insectes sociaux ${ }^{6}$. Pour comprendre en quoi le terme de cognition sociale peut se voir attribuer un sens qui est spécifique aux sociétés humaines, donnons un exemple typique de cognition distribuée

4 Bernard Conein, Les sens sociaux, Paris, Economica, 2005.

Edwin Hutchins, Cognition in the wild, Cambridge, MA, MIT Press, 1995.

- Eric Bonabeau et Guy Theraulaz, Intelligence collective, Paris, Hermès, 1994. 
qui n'est pas un cas de cognition sociale : la régulation de la température au sein d'une ruche.

Une ruche, pour bien fonctionner, nécessite une certaine régulation thermique interne. Pour résoudre ce problème, la Nature a trouvé un système ingénieux qui s'appuie sur le traitement distribué de l'information portant sur la température ambiante. Lorsque la température extérieure augmente, certaines abeilles se mettent à battre des ailes de manière à créer une ventilation à l'intérieur de la ruche. Mais les abeilles ne sont pas toutes également sensibles à la température et disposent de seuils différents à partir desquels elles vont se mettre à battre des ailes. Il en résulte un recrutement progressif des abeilles à la tâche de ventilation lorsque la température augmente qui dépend de la distribution des seuils dans la population. Cette distribution de seuils est déterminée génétiquement. Ce fonctionnement, qui permet une régulation de la température tout en n'immobilisant que le nombre d'abeilles nécessaire, est une adaptation de l'espèce à son environnement.

Dans cet exemple, nous pouvons caractériser la relation entre comportements individuels et comportements collectifs de la manière suivante :

i. chaque agent est sensible à un certain ensemble de paramètres de son environnement physique (ici la température), cette sensibilité étant une caractéristique intrinsèque de l'agent,

ii. chaque agent peut utiliser une information sur les variations de ces paramètres pour initier des actions qui vont influencer en retour la valeur de ces paramètres?

iii. l'action conjuguée des différents sujets, médiatisée par une variable d'environnent à portée collective (ici la température), a des effets dans l'environnement qui se réalisent à un niveau macro par rapport aux sujets (ici la régulation de la température globale moyenne de la ruche). Ce sont souvent ces effets que l'on qualifie de «phénomènes collectifs émergents $»$.

On parlera de « rétroaction négative » lorsque cette action va à l'encontre de cette variation, comme c'est le cas dans l'exemple présenté ici, et de "rétroaction positive » lorsque cette action amplifie cette variation (comme c'est le cas, par exemple, dans la construction des termitières). 
Deux distinctions importantes sont à relever entre l'exemple précédent et ce qui nous semble proprement humain dans le fonctionnement de nos sociétés ${ }^{8}$ :

i. Les variables d'environnement auxquelles sont sensibles les agents dans l'exemple précédent sont des caractéristiques intrinsèques des agents. Dans les sociétés humaines, celles-ci sont également le fruit d'une évolution culturelle.

ii. Dans l'exemple précédent, les variables d'environnement correspondent à des phénomènes physiques. Les autres sujets ne sont pris en compte que dans la mesure où leur présence a un effet physique sur l'environnement. En ce qui concerne les êtres humains, ce n'est pas être polémique que de dire que les individus prennent également en compte ce que sont les partenaires de leurs interactions en termes de croyances, d'intentions, de valeurs, etc. 'et que l'influence sociale agit également à ce niveau de la constitution des individus.

Dans ces deux distinctions résident plusieurs grandes questions de la sociologie à savoir : Quelle est la relation entre individus et structures collectives? Quelle est la part du social dans la constitution de l'individu? Quelle est l'influence de l'individu sur les structures collectives? Dans quel sens et dans quelle mesure sommes-nous influencés à travers les liens sociaux que nous entretenons dans les décisions que nous prenons envers nous-mêmes et envers les autres?

Ces questions sont étroitement liées au fait que, chez l'homme, plus que chez toute autre espèce animale, les nouveau-nés sont fondamentalement incomplets à leur naissance. Toutes les histoires d'enfants sauvages ont suffisamment intrigué notre imaginaire pour attirer notre attention sur le fait que les déterminants biologiques ne jouent qu'un rôle partiel dans le futur développement du nouveau-né en tant qu'être social. Cette indétermination

* Mentionnons, au passage, une littérature très intéressante en éthologie comparée où il est montré que les germes de ce qui nous semble proprement humain se retrouvent dans certaines sociétés animales évoluées, notamment chez certains primates et certaines espèces de cétacés. Voir par exemple les excellents ouvrages de Dominique Lestel, Les origines animales de la culture (Paris, Flammarion, 2001) et de Michael Tomasello, The cultural origins of buman cognition (Cambridge (MA), Harvard University Press, 1999).

9) Pour des exemples d'études expérimentales sur l'influence des intentions dans l'interaction, voir Matthew Rabin, "A perspective on psychology and economics", European economic revien, vol. 46, 2002, p. 657-685. 
partielle de l'Être humain a été maintes fois soulignée par'Tarde et est l'une des principales remarques motivant sa théorie sociologique :

La fonction cérébrale, l'esprit, se distingue des autres fonctions en ce qu'elle n'est pas une simple adaptation à une fin précise par un moyen précis, mais une adaptation à des fins multiples et indéterminées qui doivent être précisées plus ou moins fortuitement par le moyen même qui sert à les poursuivre et qui est immense, à savoir par l'imitation $[. . .]^{10}$.

Ce court passage condense de manière extraordinaire la problématique que nous avons exposée plus haut. Tarde y défend l'idée que les fins guidant un individu dans sa vie sociale sont elles-mêmes le produit de cette vie en société mais ne préexistent pas l'activité collective des individus. Elles s'élaborent chemin faisant. Tarde suggère ainsi que ces fins qui conditionnent le traitement de l'information au niveau individuel sont le produit de l'enchevêtrement au niveau collectif de ces mêmes traitements de l'information. Cet enchevêtrement instancie la boucle réflexive des fins sur elles-mêmes leur permettant de s'auto-constituer. Tarde donne son origine : l'imitation. La dynamique que ce mode de fonctionnement engendre est précisément ce que nous avons désigné sous le terme de "cognition sociale ». Nous pouvons alors proposer une définition de la cognition sociale au sein d'une population d'agents : dynamique sous laquelle la distribution ${ }^{11}$ des fins, croyances et représentations des agents composant une société se modifie sous l'effet de leurs usages ${ }^{12}$.

\section{Imitation : trait d'union entre individu et collectif}

Sans s'attarder sur des aspects généraux de l'imitation, soulignons que cette notion, contrairement à une vision populaire, est bien différente de la notion de conformisme. La première est un principe, la seconde est une loi de comportements, elles n'ont pas le même degré de généralité.

Par ailleurs, si nous allons nous concentrer ici sur les phénomènes mimétiques, il ne s'agit pas de donner l'exclusivité à l'imitation dans

10 Gabriel Tarde, Les lois de l'imitation, Les classiques des Sciences Sociales, Université du Québec à Chicoutimi, Jean-Marie Tremblay, [1890] 2002, Chapitre III, p. 61.

11 Le terme distribution doit être entendu au sens mathématique, c'est-à-dire proportions des différents types d'occurrences du phénomène considéré.

12 Une définition plus formalisée se trouve dans David Chavalarias, Métadynamiques en cognition sociale, thèse de doctorat, École polytechnique, Paris, 2004, p. 193. 
l'organisation des conduites humaines ou de prendre la position extrême de Tarde suivant laquelle « tout n'est socialement qu'inventions et imitations $^{13} \gg$. Certains comportements sont innés, d'autres acquis. Les comportements acquis dépendent de processus de décision qui résultent d'un apprentissage. Sans entrer dans les détails, les processus de prise de décision chez les êtres humains dépendent de deux grandes catégories d'apprentissage : l'apprentissage individuel et l'apprentissage social. L'imitation, quelle que soit la définition que l'on en adopte, recouvre une large part de l'apprentissage social. Les processus mimétiques se répartissent eux-mêmes en deux catégories : les processus automatiques ou inconscients qui sont présents dès la naissance chez le nouveau-né ${ }^{14}$ et peuvent prendre la forme à l'âge adulte d'un certain conformisme ; les processus réfléchis, que l'on qualifie souvent d'imitation rationnelle et que $\operatorname{Tarde}^{15}$ attribuait à des «causes logiques ». Il est entendu qu'une théorie de l'action doit prendre en compte ces différents types de prise de décision, mimétiques ou non.

Dans ce qui suit, nous nous concentrerons sur les phénomènes mimétiques en les isolant des autres types d'apprentissage dans le but d'identifier clairement les réponses qu'ils peuvent apporter aux questions que nous nous sommes posées. Plus particulièrement, nous nous intéresserons aux « causes logiques de l'imitation ».

\subsection{Approches historiques de l'imitation}

L'imitation a été considérée dans beaucoup de systèmes épistémologiques comme un lieu de démarcation entre sociétés humaines et autres sociétés animales. On lit par exemple chez Aristote (Poétique 4) : «L'Homme diffère des autres animaux en ce qu'il est plus apte à l'imitation ». À la fin du XIX siècle, les sciences humaines et sociales ont connu un engouement sans précédent pour l'imitation en tant que principe structurant commun à la cognition individuelle et à la cognition sociale. Alors que James Marc Baldwin $^{16}$, un des pères de la psychologie expérimentale, plaçait l'imitation comme clé de voûte de sa théorie du développement et notamment de

13 Gabriel Tarde, op. cit., p. 21.

${ }_{14}$ Andrew Meltzoff et Wolfgang Prinz (dir.), The imitative mind: Development, evolution, and brain bases, Cambridge, Cambridge University Press, 2002.

15 Gabriel Tarde, op. cit., Chapitre V.

16 James Mark Baldwin, Le développement mental chez l'enfant et dans la race, Paris, Alcan, 1897. 
l'émergence de la volition, Gabriel Tarde promettait aux lois générales qui régissent la répétition imitative un rôle analogue en sociologie à celui de l'hérédité en biologie.

Ces deux approches se rejoignaient autour de deux convictions d'une étonnante actualité, dont la portée épistémologique est de première importance pour les sciences sociales :

i. Comme le défend Tarde ${ }^{17}$, une théorie du social se doit de se doter de ses propres outils conceptuels et formels. Pour cela il serait trompeur de chercher à donner aux sciences sociales un " air biologique " ou un " air mécanique » afin de récupérer les outils formels d'autres disciplines car ce serait « lâcher la proie pour l'ombre ». Autrement dit, les dynamiques sociales sont fondamentalement irréductibles aux dynamiques gouvernant les systèmes naturels ${ }^{18}$. La raison principale de cette conviction, pour Tarde comme pour Baldwin, étant que ces dynamiques sont par essence d'origine mimétique.

ii. Il y a dans les systèmes sociaux co-détermination des individus et du collectif et elle est possible par l'imitation. Les systèmes sociaux engendrent ainsi de façon interne leur propre espace de signification qui est la projection au niveau collectif d'un processus dialectique intrinsèquement social de constitution du sens. Ce processus s'initie dès l'émergence de la distinction entre soi et autrui : « My sense of myself grows by imitation of you, and my sense of yourself grows in terms of my sense of myself ${ }^{19}$ \%.

Le premier point est important dans une approche heuristique car il pose des contraintes sur les types de modèles, formels en particulier, qui sont susceptibles de nous éclairer sur les faits sociaux. Avec notre recul historique, cette intuition est renforcée par les tentatives infructueuses de l'économie " orthodoxe » et de la sociobiologie pour expliquer certains faits sociaux, insuccès révélés en particulier par leurs difficultés à rendre compte de la diversité des fins (ou des préférences).

Le deuxième point est tout aussi important car c'est un programme de recherche qui se déploie entre individualisme méthodologique et holisme,

17 Gabriel Tarde, op. cit., Introduction au chapitre I.

18 Mème si des réductions partielles n'en restent pas moins instructives.

19 James Mark Baldwin, op. cit. 
préfigurantl'«individualisme méthodologique complexe ${ }^{20} »$. Les alternatives qu'il propose peuvent constituer des apports substantiels aux débats qui font rage entre ces deux prises de position. En particulier, il y avait à l'époque de 'Tarde l'idée que l'imitation délibérée est le premier signe distinctif de la volition et de l'action raisonnée au cours du développement : la manière dont l'individu se démarque de son environnement social tient précisément à une modification de la forme initialement innée de l'imitation ; c'est donc un espace de libre choix créé à l'intérieur même du domaine réservé aux processus de détermination sociale.

\subsection{L'imitation aujourd'hui : entre holisme et individualisme méthodologique}

De manière étonnante, ce programme de recherche n'a pas trouvé de successeur immédiat. Après avoir été quelque peu délaissé, le thème de l'imitation a cependant été repris par de grandes figures des sciences sociales : Piaget en psychologie, Girard (1961) en anthropologie, Keynes et Hayek en économie ${ }^{21}$; pour être aujourd'hui un sujet d'une actualité brûlante aussi bien en psychologie sociale ${ }^{22}$, qu'en anthropologie évolutionniste $^{23}$, en économi ${ }^{24}$, en éthologi ${ }^{25}$, en sociologie ${ }^{26}$; et également

20) Jean-Pierre Dupuy et Paul Dumouchel (dir.), L'auto-onganisation, de la physique an politique, Paris, Seuil, 1983.

21 Jean-Pierre Dupuy, «Economic as symptom », dans Transforming economics. Perspectives on the critical realist project, Londres et New York, Routledge, 2004, p. 227-251.

22 Albert Bandura, Social learning theory, Englewood Cliffs (NJ), Prentice-Hall, 1977.

2.3 Boyd, Robert et Peter J. Richerson, Culture and the evolutionary process, Chicago, Chicago University Press, 1985 ; Merlin Donald, Origins of the Modern Mind, Cambridge (MA), Harvard University Press, 1991 ; Joe Henrich et Richard Boyd, « The evolution of conformist transmission and the emergence of between-group differences », Evolution and buman behavior, vol. 19, n" 4, 1998, p. 215-241.

24 André Orléan, «Les interactions mimétiques », dans Jacques Lesourne, André Orléan et Bernard Walliser (dir.), Lecons de microéconomie évolutionniste, Paris, Odile Jacob, 2002, p. 119-157; Reinhard Selten et Jose Apesteguia, «Experimentally observed imitation and cooperation in price competition on the circle», Games and economic behavior, vol. 51, n' 1, 2005, p. 171-192.

25 Richard Byrne et Anne Russon, «Learning by imitation: A hierarchical approach », Behavioral and brain sciences, vol. 21, $\mathrm{n}^{\circ}$ 5, 1998, p. 667-684 ; Michael Tomasello, The cultural origins of buman cognition, Cambridge (MA), Harvard University Press, 1999.

26 Peter Hedstrom, «Rational Imitation », dans Peter Hedstrom et Richard Swedberg (dir.), Social mechanism. An analytical approach to social theory, Cambridge, Cambridge University Press 1998, p. 306. 
en psychologie, neurologie, robotique ou philosophie de l'esprit ${ }^{27}$. Certaines particularités de l'imitation humaine ont ainsi pu être mises en évidence, même si le consensus n'est pas absolu, telles que la relation entre les représentations de soi et d'autrui, l'inférence de fins à partir de l'observation des moyens ou l'imitation des composantes procédurales liées à l'action.

Mais force est de constater qu'en traversant les disciplines, le thème de l'imitation a perdu son originalité première, chaque théorie ne déployant qu'un aspect de l'imitation. Ceci est particulièrement visible en économie où certains chercheurs comme André Orléan (dans ses premiers travaux) ou Jean-Pierre Dupuy ont pu privilégier une approche girardienne des processus mimétiques, opaques aux individus, qui rappelle ce que Baldwin nommait imitation simple et que Tarde rangeait dans les imitations relevant de causes « non logiques »; alors que d'autres ${ }^{28}$ privilégient une approche rationnelle de l'imitation, dans le prolongement de la notion d'imitation persistante chez Baldwin et de l'imitation relevant de causes logiques chez Tarde.

D'un côté, nous avons l'idée qu'un individu désire parce qu'il imite, de l'autre celle qu'il imite parce qu'il désire. Formellement, cela se traduit par des approches très différentes. Pour le courant que l'on pourrait qualifier de girardien, la valeur des choses, des opportunités d'action se lisent dans les désirs des autres. L'imitation est alors en quelque sorte un conformisme englobant le niveau des désirs ou des fins, chacun recherchant ce qu'il pense deviner chez l'autre. Mais le fait qu'un individu ait telle ou telle structure sur ses désirs ne change pas la manière dont il subira cette influence mimétique. La manière dont un individu choisit ses modèles, la forme de l'imitation, est une propriété commune à tous les individus. En revanche, pour le courant qui s'attache à l'aspect logique et rationnel de l'imitation, celle-ci est au service de principes ou de fins pré-établis : A imite B (en adoptant sa manière d'agir ou sa stratégie) s'il estime que ce dernier est particulièrement performant suivant des critères reconnus par $A$, ces critères étant une propriété permanente de A (par exemple, si A est un individu cupide, il aura

27 Voir, par exemple, Jacqueline Nadel et Jean Decety (dir.), Imiter pour découvrir l'bumain. Psychologie, neurologie, robotique et philosophie de l'esprit. Psychologie et science de la pensée, Paris, PUF, 2002.

28 Reinhard Selten et Axel Ostmann, «Imitation Equilibrium », Homo oeronomicus, vol. 18, 2001, p. 111-149 ; Joshua Frank, « Natural selection, rational economic behavior, and alternative outcomes of the evolutionary process ", Joumal of socio-economics, vol. $32, n^{\prime \prime} 6,2003$, p. 601-622. 
certainement une propension à imiter particulièrement des individus qui se seront fait remarquer par leur opulence). La forme de l'imitation est alors une caractéristique individuelle.

Outre le fait que ces approches partielles de la notion d'imitation ont pour conséquences des débats interminables sur la définition même d'imitation, le fait de ne considérer qu'une seule face de la médaille rompt le lien établi par l'imitation entre cognition individuelle et cognition sociale, faisant disparaitre du même coup cette possibilité de voie médiane entre individualisme méthodologique et holisme.

\subsection{Jeux métamimétiques}

L'étude de l'imitation semble donc ouvrir une voie vers cette « dialectique récursive » par laquelle «la pensée des moyens de la fin (devient) le moyen de repenser les fins ${ }^{29}$ \%. Pour autant, le cadre conceptuel posé par Tarde reste flou sur les moyens à mettre en oeuvre en pratique pour saisir par des modèles de pensée les propriétés qualitatives qu'apportent ce mode d'autotransformation du social. Les arguments qu'il utilise sont essentiellement des descriptions de phénomènes. Il leur manque comme complémentaires des reconstructions de faits stylisés ou des ébauches de modèles qui nous permettraient de penser plus précisément la relation entre individus et collectif. Tarde nous fournit cependant un indice dans la définition qu'il donne des causes logiques de l'imitation:

Les causes sociales sont de deux sortes : logiques ou non logiques. [...] Les causes logiques agissent quand l'innovation choisie par un homme l'est parce qu'elle est jugée par lui plus utile ou plus vraie que les autres, c'est-àdire plus d'accord que celles-ci avec les buts ou les principes déjà établis en lui (par imitation toujours) ${ }^{30}$.

La quasi-totalité de cette citation correspond à peu de choses près à la description de l'imitation rationnelle que l'on peut attendre de la part d'un économiste : un individu choisit une action plutôt qu'une autre, choisit d'imiter untel plutôt que tel autre, parce que le résultat attendu de cette imitation correspond à la meilleure évaluation sur l'échelle de ses préfé-

29 Jean-Louis Le Moigne, "Représenter et raisonner les comportements socioéconomiques ", dans Christine Roland-Lévy et Philippe Adair (dir.), Psychologie économique, théorie et applications, Paris, Economica, 1988, p. 317-337.

30 Gabriel Tarde, op. cit, Chapitre V, p. 106. 
rences. Mais 'Tarde rajoute, sans cependant s'étendre, «par imitation toujours ", suggérant ainsi que les buts et les principes des individus euxmêmes évoluent suivant une dynamique mimétique.

Mais, dans ce cas, quels sont les buts et les principes qui servent de référence lorsqu'il s'agit de changer par imitation ses propres buts et principes? Tarde ne semble pas suggérer que cette seconde imitation au niveau meta soit de nature différente de celle qui agit au niveau des actions (par exemple qu'elle relèverait de causes non logiques, ce qui nous rapprocherait de Girard). Il suggère plutôt que l'on doit dissocier le «principe d'imitation des critères » guidant les actes d'imitation, ces derniers pouvant également faire l'objet d'un acte d'imitation.

Cette approche trouve une illustration dans le formalisme des jeux métamimétiques $^{31}$. Celui-ci s'appuie sur les relations possibles entre la spécificité de l'imitation humaine et certaines capacités cognitives propres aux êtres humains telles que la réflexivité ou la métacognition. En particulier, cela permet de formaliser cette dissociation entre principe d'imitation et critères d'imitation. L'imitation d'un nouveau moyen pour satisfaire une ancienne fin devient alors le principal facteur d'adoption de nouvelles fins.

D'un point de vue formel, on montre ainsi que les règles d'imitation peuvent être leurs propres métarègles : une règle d'imitation, en tant que processus permettant de sélectionner un modèle, puis un trait à copier, peut être utilisée pour choisir, parmi les modèles possibles, celui auquel un agent empruntera sa nouvelle règle d'imitation. Cette équivalence d'un niveau et de son meta niveau " quelque part » dans la description de la dynamique rejoint les caractéristiques fondamentales des systèmes capables d'autotransformation telles qu'elles ont pu être décrites par des auteurs comme Hofstadter ${ }^{32}$.

Déclinée à travers le prisme de l'hétérogénéité des agents, cette représentation formelle de l'imitation permet de rendre compte d'une autoorganisation des fins au niveau d'une population, au sens de sélection spontanée dans l'ensemble des fins imaginables par les agents d'une distribution de fins particulières. Les configurations sociales stables dans ce cadre correspondent à un nouveau concept d'équilibre, les « états contre-

31 David Chavalarias, op. cit.

32 Douglas Hofstadter, Gödel, Escher, Bach: An eternal golden braid, s. l., New York (NY), Basic Books, 1999. 
factuellement stables » : " aucun individu ne peut s'imaginer mieux qu'il n'est en se mettant contrefactuellement à la place d'autruij ${ }^{33} »$. Le chemin que parcours un système pour arriver à ce type d'équilibre (ou d'attracteurs, le cas échéant) correspond précisément à ce que nous avons appelé plus haut un processus de « cognition sociale ».

Cette approche des dynamiques sociales se révèle en rupture avec les approches formelles traditionnelles qui se placent généralement dans un cadre finaliste où les agents optimisent une quantité qui préexiste à leur activité. Elle interprète en effet l'hétérogénéité auto-organisée des systèmes sociaux humains comme une différenciation par co-évolution d'une multiplicité de critères possibles, qui dès lors ne peuvent se concevoir que de manière contextuelle et d'un point de vue individuel ${ }^{34}$.

\section{Représentation de la cognition sociale autour d'un jeu métamimétique}

\subsection{Comment représenter l'auto-transformation des fins}

De même que le cercle griffonné sur une feuille de papier permet de penser les propriétés du cercle bien qu'il n'en ait quasiment aucune, nous exposerons maintenant les grandes lignes d'un modèle multi-agents susceptible d'illustrer les propriétés d'auto-transformation et les relations individus-collectif que nous avons ébauchées. Soulignons qu'il ne s'agit pas de dresser un portrait réaliste des systèmes sociaux, mais de s'intéresser à des types de systèmes tels que les relations entres les différents niveaux d'organisation qu'ils mettent en scène nous permettent de réfléchir aux relations entre les différents niveaux d'organisation que nous identifions au

David Chavalarias, op. cit.

Nous renvoyons le lecteur intéressé par plus de précisions aux travaux antérieurs : David Chavalarias, "Metamimetic games: Modeling metadynamics in social cognition " (Joumal of artificial societies and social simulations, vol. 9, $\mathrm{n}^{\circ} 2,2006$, http://jasss.soc.surrey.ac.uk/9/2/5.html), pour un exposé plus détaillé du cadre formel des jeux métamimétiques et David Chavalarias, Chavalarias, David, "Cooperation as the outcome of a social differentiation process in metamimetic games » (dans Bruce Edmonds, Cesáreo Hernández lglesias et Klaus G. Troitzsch (dir.), Social simulation: technologies. Advances and new discoveries, IDEA, 2007, à paraître), pour un exemple d'application à l'émergence de la coopération. Nous ne fournirons ici qu'un exemple minimal pour montrer en quoi ce type d'approche peut nous aider à penser l'auto-transformation du social en termes de cognition sociale. 
sein des systèmes sociaux. Il s'agit donc, pour suivre une distinction introduite par Isabelle Stengers, de proposer un modèle de concepts plutôt qu'un modèle de la réalité.

Revenons sur cette « adaptation à des fins multiples et indéterminées qui doivent être précisées plus ou moins fortuitement par le moyen même qui sert à les poursuivre [...] à savoir l'imitation ${ }^{35}$ ». Si nous prenons Tarde à la lettre, le modèle le plus simple que nous pouvons imaginer des transformations des fins est de supposer qu'une fin est modifiée au cours d'un processus d'imitation où elle intervient elle-même comme critère dans l'acte d'imitation. Elle est alors à la fois l'objet de la transformation et son motif. Par exemple, il se peut très bien qu'afin de pouvoir faire de la recherche sur des thèmes de prédilection (fin 1), il semble incontournable de recueillir soimême des financements (fin 2), cette seconde fin finissant par occuper en tâches administratives tout le temps initialement consacré à la recherche. Ce type de glissement est fort fréquent. Nous réfléchirons maintenant à l'une de ses schématisations dont la simplicité aura l'avantage de nous permettre d'appréhender les phénomènes collectifs. Ce glissement peut se caricaturer de la manière suivante : « si au regard de la fin 1 que je poursuis je m'aperçois que poursuivre la fin 2 est l'une des meilleures solutions pour arriver à mes fins, alors je vais consacrer du temps à poursuivre cette seconde fin ». Ici, le verbe « apercevoir » rend compte d'un apprentissage. Dans le cadre que nous nous sommes fixé, celui-ci est d'ordre mimétique. Ceci nous permet de réinterpréter la phrase de la manière suivante : « si en me mettant contrefactuellement à la place de l'une de mes connaissances, je me trouve dans une situation meilleure relativement aux fins que je poursuis actuellement, je vais chercher à m'inspirer du comportement ou des fins de cette personne ». La question est maintenant de savoir ce qui se passe lorsque toute une population raisonne de la sorte. Certains individus vont-ils se stabiliser sur des fins particulières? Assistera-t-on à l'émergence de groupes partageant des fins similaires? Quels types de dynamiques obtient-on sur les fins et quels types de relations individus/collectif cela implique-t-il?

\subsection{Représentation stylisée d'une situation d'interaction sociale}

L'une des manières de répondre à ces questions est de considérer effectivement un ensemble d'agents raisonnant de la sorte, capables

35 Référence déjà donnée à la note infrapaginale 10. 
d'imaginer et de suivre un ensemble circonscrit de fins et d'actions, puis d'en étudier les dynamiques collectives. Il nous faut pour cela imaginer une situation stylisée d'interaction sociale : un cadre d'interaction et l'ensemble des représentations que peuvent s'en faire les agents.

Nous nous placerons pour cet exemple dans le cadre de la théorie des jeux et nous prendrons le modèle d'interaction le plus simple et le plus répandu dans ce domaine. Il consiste en une interaction dyadique et répétée entre deux joueurs. À chaque période, les joueurs peuvent opposer deux comportements distincts à leurs partenaires, que nous désignerons par $\mathrm{C}$ et D. Nous considérerons un cas particulier où l'interaction se traduit, pour chacun des joueurs, par des gains ou des pertes matérielles (le plus classique étant d'imaginer des gains monétaires) :

i. si chacun des agents choisit $C$, le gain individuel est de $R$,

ii. si chacun des agents choisit $\mathrm{D}$, le gain individuel est de $P$,

iii. s'ils choisissent des actions différentes, celui qui a choisi $\mathrm{D}$ gagne $T$ et celui qui a choisi $C$ gagne $S$.

Cette structure de jeu peut se résumer par une matrice d'allure bien connue des théoriciens des jeux (Table 1).

L'étude que nous présentons ici porte sur un jeu célèbre appelé dilemme du prisonnier qui traduit le type de conflit qu'il peut y avoir entre intérêts individuels et intérêt collectif. La seule caractéristique dont nous aurons besoin, cependant, dans cette brève analyse ${ }^{36}$ est le fait que, quelle que soit l'action de son partenaire, un joueur a des gains supérieurs s'il joue D que s'il joue $\mathrm{C}$.

\section{Table 1}

Matrice donnant la structure de l'interaction sociale stylisée en terme de gains matériels

\begin{tabular}{|c|c|c|}
\hline Joueur A & \multicolumn{2}{|c|}{ Joueur B } \\
\hline & $\mathrm{C}$ & $\mathrm{D}$ \\
\hline $\mathrm{C}$ & $\mathrm{R}, \mathrm{R})$ & $(\mathrm{S}, \mathrm{T})$ \\
\hline $\mathrm{D}$ & $(\mathrm{T}, \mathrm{S})$ & $(\mathrm{P}, \mathrm{P})$ \\
\hline
\end{tabular}

36 Pour une étude plus détaillée, voir David Chavalarias, "Cooperation as the outcome of a social differentiation process in metamimetic games ", ibid. 
Une fois défini le type d'interaction, il nous reste à décrire la structure du réseau social que nous étudierons et les représentations que peuvent se faire les agents de ces interactions.

Nous placerons les agents sur une grille carrée torique ${ }^{37}$, ces agents ayant la possibilité d'interagir avec leurs huit voisins (figure 1).

\section{Figure 1}

Un agent avec ses huit voisins (voisinage de Moore)

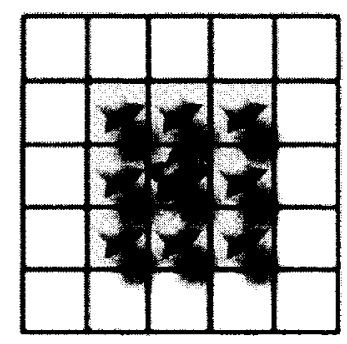

En ce qui concerne les représentations que se font les agents de leurs interactions, celles-ci dépendront, comme nous l'avons déjà mentionné, des catégories perceptives dont disposent les agents et de la manière dont ils les agencent pour se représenter leurs fins. Nous ne nous intéresserons pas ici à la manière dont se forment ces catégories perceptives, qui est un autre problème d'évolution culturelle, mais uniquement à la manière dont s'autoorganisent les fins possibles. Nous ferons donc les hypothèses suivantes :

i. les agents peuvent percevoir les gains des partenaires avec lesquels ils interagissent,

ii. les agents peuvent percevoir le comportement et les fins de leurs partenaires.

En ce qui concerne les opérations que peuvent faire les agents sur ces perceptions élémentaires, nous supposerons que :

i. les agents peuvent calculer les fréquences de comportements et de fins parmi leurs partenaires, correspondent. 
ii. les agents peuvent calculer le maximum de deux quantités,

iii. les agents peuvent calculer le minimum de deux quantités.

Cela nous donne quatre fins possibles :

i. être l'agent dont les gains sont les plus élevés dans le voisinage,

ii. être l'agent dont les gains sont les moins élevés dans le voisinage,

iii. être dans la majorité relativement aux comportements et aux fins,

iv. être dans la minorité relativement aux comportements et aux fins.

Traduit en termes de règles d'imitation, cela nous donne quatre règles possibles que l'on retrouve souvent dans la littérature des jeux évolutionnaires (table 2):

i. Maxi : «copie l'agent de ton voisinage dont les gains sont les plus élevés »,

ii. Mini : « copie l'agent de ton voisinage dont les gains sont les moins élevés »,

iii. Conformisme: «copie la règle ou le comportement majoritaire parmi tes voisins ",

iv. Anticonformisme : « copie la règle ou le comportement minoritaire parmi tes voisins $»$.

Nous attirons l'attention sur le fait que l'agent étant dans son propre voisinage, il ne s'engagera pas dans un acte d'imitation s'il est effectivement l'un des " meilleurs " agents de son voisinage. On dira alors qu'il est « contrefactuellement stable ».

Table 2

L'espace des fins possibles traduites en termes de règles d'imitation

\begin{tabular}{|c|c|c|}
\hline Opérateurs & \multicolumn{2}{|c|}{ Perceptions } \\
\hline & Fréquences & Gains \\
\hline $\max$ & Conformisme & Maxi \\
\hline $\min$ & Anticonformisme & Mini \\
\hline
\end{tabular}

La particularité des jeux métamimétiques est de considérer que les règles d'imitation interviennent aussi bien dans la modification des comportements que dans celle des règles d'imitation elles-mêmes. Considérons par exemple un agent maxi qui se demanderait quel est le meilleur moyen de parvenir à ses fins (c'est-à-dire de maximiser ses gains). Il se peut qu'en regardant autour de lui il se rende compte que les individus qui réussissent le mieux d'un point de vue matériel sont conformistes. Il pourra alors se 
dire "puisque c'est ce qui marche le mieux, à partir de maintenant je vais faire comme tout le monde ». Dans ce cas-là, la règle de maximisation des gains se placera en position meta par rapport à une nouvelle règle conformiste, considérée de ce point de vue comme un moyen (figure 2).

Figure 2

Un agent maxi peut adopter temporairement une attitude conformiste tout en gardant en tête que ceci n'est qu'un moyen pour maximiser ses gains.

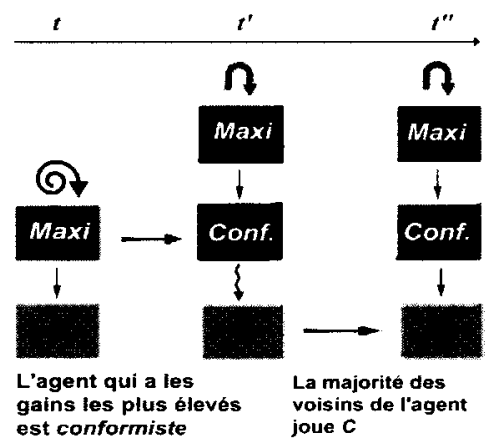

Mais une autre chose peut se produire, à savoir que notre agent maxì à force de jouer au conformiste, le devient réellement, soit par habitude, soit par plaisir, soit parce que, cognitivement, il ne peut pas maintenir plusieurs buts en même temps (figure 3).

\section{Figure 3}

Un agent maxi peut adopter une attitude conformiste en oubliant que ce n'était qu'un moyen pour satisfaire une ancienne fin.

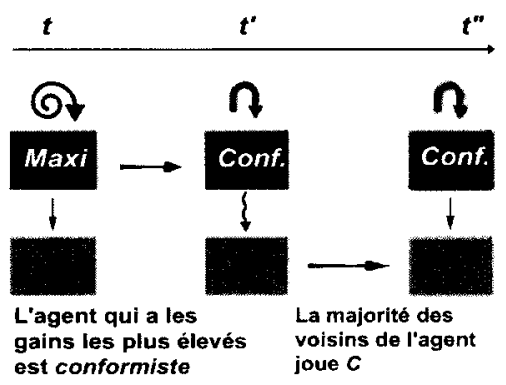


Ce dernier cas est un exemple du type de substitution de fins par des moyens dont nous parlions à la fin de la section 3.1. Illustrons maintenant par une étude computationnelle ce qui se passe lorsque toute une population se comporte comme dans l'exemple de la figure 3.

\subsection{Cognition sociale : quand le changement va changeant}

Autant nous pouvons imaginer ce qui se passe lorsque deux agents jouent à un jeu métamimétique, autant cela dépasse nos capacités de représentation lorsque la population comporte des milliers d'agents. Si nous voulons étudier les relations individus/collectif, il nous faut passer par un médium externe ou une représentation analytique. Nous avons choisi de recourir aux simulations multi-agents et nous présentons ici l'effet de comportements métamimétiques à l'échelle d'une population de 10000 agents en réalisant in silico (figure 4) la situation stylisée d'interaction sociale que nous venons de décrire (figure 3). La structure stylisée d'interaction sociale que nous avons prise pour cet exemple a peu d'importance relativement aux phénomènes que nous voulons illustrer. Rappelons cependant qu'il s'agit d'un dilemme du prisonnier et dans ce cas précis nous avons considéré l'exemple donné par Axelrod ${ }^{38}(T=5, R=3, \quad P=1, S=0)$. Pour une distribution initiale uniforme des règles d'imitation, les structures observées sont robustes à la fois vis-à-vis des paramètres de la matrice (pour autant que l'on reste dans la même classe de jeux) et vis-à-vis de la distribution initiale de comportements ${ }^{39}$. Dans le cadre des études sur l'émergence de la coopération et contrairement à beaucoup de modèles évolutionnaires, cette approche permet d'interpréter la coopération sous l'angle de la différenciation sociale dans des termes bien différents d'une différenciation des comportements par le jeu d'interactions entre « agents rationnels égoïstes».

Regardons donc ce que donne une simulation d'une population d'agents métamimétiques tel que nous venons de le décrire (voir figure 4). Plusieurs choses sont frappantes aux premiers abords et d'étroits parallèles peuvent être tracés avec la pensée de Tarde.

38 Robert Axelrod, The Evolution of Cooperation, New York, Basic Books, 1984.

39) Voir David Chavalarias, op. cit., 2007. 

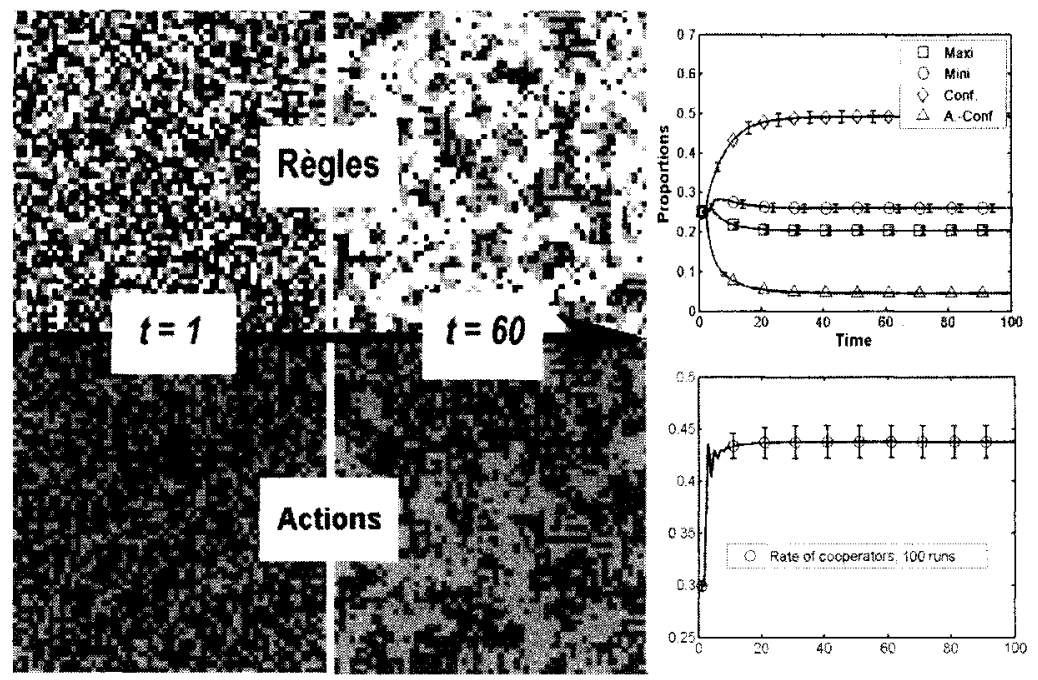

Fvolution de la distribution spatiale des règles d'imitation (partie supérieure) et des comportements (partie inférieure) à partir d'un état initial désordonné vers un attracteur structuré. Chaque petit carré représente un agent. Chaque agent interagit avec ses huit voisins comme sur la figure 1. La configuration à l'attracteur est globalement stable à l'exception de quelques agents qui ne cessent de changer leur règle ou leur comportement. Code couleur : pour les règles - blanc : les conformistes, noir : les anti-conformistes, gris clair : mini, gris foncé : maxi. Pour les comportements - gris clair : action C, gris foncé : action D. La population comporte ici 6400 agents. Dans l'état initial de gauche, les règles sont assignées aux agents de manière aléatoire uniforme, les comportements sont assignés de manière aléatoire en respectant une proportion initiale de $30 \%$ de comportements C. Les graphiques à droite montrent l'évolution des distributions des différents types de traits au cours du temps. Ies barres d'erreur représentent la variance calculée sur 10 simulations indépendantes. On peut constater qu’à $t=60$, la dynamique a bien atteint son attracteur. 


\subsubsection{Auto-cohérence des règles d'imitation}

[La logique de l'imitation conduit à ce que chaque homme] se met finalement d'accord avec lui-même par l'adoption d'un système logique d'idées et d'une conduite conséquente ${ }^{40}$.

'Tout d'abord, après une courte période d'intense réorganisation (moins de 20 pas de temps) pendant laquelle la plupart des agents changent leur règle et leur comportement, la population finit par se stabiliser dans un état quasi statique (attracteur) où seulement quelques agents continuent de changer périodiquement leur règle ou leur comportement. Dans cet état, la plupart des agents sont contrefactuellement stables : ils sont en équilibre avec leur environnement par rapport aux règles qu'ils ont adoptées.

\subsubsection{Groupes sociaux}

De là cette définition du groupe social : une collection d'êtres en tant qu'ils sont en train de s'imiter entre eux ou en tant que, sans s'imiter actuellement, ils se ressemblent et que leurs traits communs sont des copies anciennes d'un même modèle $e^{41}$.

Observons ensuite que cet état collectif est fondamentalement " hétérogène ». Contrairement à une croyance répandue selon laquelle l'imitation mène à l'uniformisation, l'imitation lorsqu'elle s'exerce au niveau meta aboutit à une différenciation. Cet état collectif est organisé en sousgroupes d'agents dont les structures sont caractéristiques des capacités cognitives dont nous avons munis les agents. Ces structures nous permettent même de deviner le code couleur de la figure 4 : les conformistes sont structurés en larges zones plus ou moins convexes à l'intérieur desquelles ils sont effectivement en majorité. À l'intérieur de ces zones, leur comportement est uniforme mais peut être indifféremment $D$ ou $C$. Les anti-conformistes sont éparpillés sur tout le territoire, ils sont isolés et globalement en minorité. Leur comportement est en général contraire à ce qui est pratiqué dans leur voisinage. Les populations d'agents maxi et mini, quant à elles, sont la plupart du temps enchevêtrées, les maxi ayant adopté l'action $D$ associée aux gains les plus forts, et les mini ayant adopté l'action

Voir Gabriel Tarde, op. cit., Chapitre IV p. 39.

41 Gabriel Tarde, op. cit., Chapitre III, p. 61. 
$C$ associée aux gains les plus faibles : les uns profitent des autres qui s'en réjouissent.

Ces « groupes sociaux artificiels » sont la projection au niveau collectif de l'espace des représentations envisageables par les agents, que Tarde désignait comme l'ensemble des «virtualités élémentaires ». Leur formation en tant que macro-structures est une conséquence des interactions mimétiques répétées entre agents au cours desquelles des représentations potentielles s'actualisent dans un contexte social.

\subsubsection{Auto-transformation du social}

Quelque chose de bien plus important qu'une simple augmentation de différence s'accomplit dans l'univers incessamment, la différenciation de la différence elle-même. Le changement même y va changeant, et dans un certain sens qui, d'une ère de différences crues et juxtaposées, comme de couleurs criardes et non fondues, nous achemine à une ère de différences harmonieusement nuancées ${ }^{42}$.

Comme nous l'avons défendu, l'imitation telle que nous l'avons présentée doit être envisagée dans les modèles comme un principe, et non comme une loi : nous n'obtenons une loi ou une règle d'imitation qu'à partir du moment où l'on définit le critère par rapport auquel doit s'effectuer l'imitation ${ }^{43}$. Cela signifie que la loi d'évolution globale du système d'agents est en fait définie par l'arrangement spatial des règles d'imitation des différents agents. La partie supérieure de la figure 4 est en quelque sorte un dessin de cette loi d'évolution à deux périodes différentes. La différence du dessin entre ces deux périodes signifie donc que cette loi d'évolution ellemême va changeant au cours de l'évolution de la société artificielle. C'est en ce sens que l'on peut dire qu'il y a auto-transformation du système au cours de son fonctionnement. Cette dynamique qui agit autant au niveau des

42 Gabriel Tarde, Les lois sociales, esquisse d'une sociologie, I.es classiques des Sciences Sociales, Université du Québec à Chicoutimi, Jean-Marie Tremblay, [1898, Paris, Alcan] 2002, p. 69.

43 Nous attirons l'attention du lecteur sur le fait que le titre du premier ouvrage de Tarde est bien Les lois de l'imitation et non La loi de l'imitation, ce qui souligne l'idée d'un principe duquel peuvent découler plusieurs types de régularités. Nous pouvons ici faire une analogie avec le principe de maximisation d'utilité espérée en économie, bien que ce dernier agisse sur des critères qui, la plupart du temps, sont supposés immuables. 
comportements qu'au niveau des règles d'évolution de ces comportements est une illustration de ce que nous avons appelé « cognition sociale ».

\subsection{Holisme ou individualisme méthodologique}

Si maintenant nous considérons cet exemple à la lumière des deux grands paradigmes des sciences sociales - individualisme méthodologique et holisme -, nous constatons qu'il ne relève ni de l'un, ni de l'autre mais d'un troisième type, l'« individualisme méthodologique complexe ». Dire que l'évolution de cette société artificielle est le produit des interactions d'individus déjà constitués hors de la société n'a aucun sens car nous voyons ici que l'identité des individus change au cours du jeu social et que leurs téléologies individuelles se constituent précisément au cours de leur évolution dans un contexte social.

Mais dire que l'évolution de cette société est dirigée par des régularités qui préexistent aux interactions des agents n'a pas non plus de sens. Certes, nous pourrions objecter que l'espace des représentations que nous avons considéré est ici donné en dehors des agents, qu'il préexiste à leur activité. Rappelons simplement que nous avons laissé de côté la constitution de cet espace pour des raisons de clarté de l'exposé. Comme le souligne Tarde dans son oeuvre, cet espace est en fait le produit de l'évolution culturelle. C'est un espace ouvert où certaines innovations individuelles sans cesse apparaissent et ne perdurent dans le contexte social que dans la mesure où elles sont reprises et amplifiées par la dynamique mimétique collective. L'adoption de nouveaux principes, de nouvelles valeurs, de nouveaux traits sociaux est conditionnée par la métadynamique définie par l'arrangement des entités sociales existantes, mais chaque individu porte en lui la possibilité d'introduire l'innovation qui un jour modifiera de manière significative cette métadynamique. Ceci est bien visible, par exemple, dans l'évolution d'une langue ou des habitudes vestimentaires.

\section{Conclusion}

Nous avons souhaité dans cet article préciser le concept de « cognition sociale » en insistant sur son caractère spécifique aux sociétés humaines en tant que processus qui aboutit à l'auto-organisation des téléologies individuelles.

Nous avons ainsi vu l'importance de considérer dans une approche des systèmes sociaux non seulement les dynamiques sur les comportements 
mais également les métadynamiques sur les règles, les croyances ou les représentations des agents qui déterminent ces comportements. Ces métadynamiques sont en effet à même d'exprimer la particularité d'autotransformation du social.

Le terme cognition est alors envisagé sous un angle qui nous rappelle la notion de " clôture opérationnelle » introduite par Francisco Varela ${ }^{44}$. Il s'agit d'un traitement de l'in-formation : les sociétés forment leurs propres constituants au cours de leur fonctionnement, l'espace des règles, croyances et représentations d'une société évolue sous l'influence de leur usage.

Comme le défendait Tarde en d'autres termes, nous avons vu que l'imitation était effectivement susceptible de rendre compte de manière très concrète de ce phénomène de cognition sociale. Dès lors, celle-ci exprime une différenciation sociale par co-évolution culturelle, la culture pouvant se définir comme «l'individuation par le collectif ${ }^{45}$ ». Elle aboutit à l'émergence de groupes sociaux et de normes, ceux-ci apparaissant pour un observateur extérieur comme des macro-régularités distinctes de la somme des activités individuelles. Un exemple simple nous a permis de montrer en quoi une approche système complexe est susceptible de nous aider à envisager ce type de processus et à appréhender «l'inséparable conjonction d'un fonctionnement et d'une transformation dans un contexte et par rapport à quelques projets eux-mêmes changeants " ${ }^{46}$.

\section{Bibliographie}

Axelrod Robert, The Evolution of Cooperation, New York, Basic Books, 1984.

Baldwin, James Mark, Le développement mental chę. l'enfant et dans la race, Paris, F. Alcan, 1897. Bandura, Albert, Social learning theory, Englewood Cliffs (NJ), Prentice-Hall, 1977.

Bonabeau, Eric et Guy Theraulaz, Intelligence collective, Paris, Hermès, 1994.

Bourgine, Paul, «What is cognitive economics? », dans Paul Bourgine et Jean-Pierre Nadal (dir.), Cognitive economics. An interdisciplinary approach, Berlin, Springer, 2004, p. 1-12.

Boyd, Robert et Peter J. Richerson, Culture and the evolutionary process, Chicago, Chicago University Press, 1985.

4 Fransisco Varela, «L'auto-organisation : de l'apparence au mécanisme », dans JeanPierre Dupuy et Paul Dumouchel (dir.), L'anto onganisation, de la physique an politique, Paris, Seuil, 1983, p. 147-164.

45 Dominique Lestel, op. cit..

46 Jean-Louis Le Moigne, op. cit. 


\section{NPSS, VOLUME 2, NUMERO 2, 2007}

Byrne, Richard et Anne Russon, "Learning by imitation: A hierarchical approach », Behavioral and brain sciences, vol. 21, n“ 5, 1998, p. 667-684.

Chavalarias, David, "Cooperation as the outcome of a social differentiation process in metamimetic games », dans Bruce Edmonds, Cesáreo Hernández Iglesias et Klaus G. Troitzsch (dir.), Social simulation: technologies. Advances and new discoveries, IDEA, 2007, à paraître.

Chavalarias, David, Métadynamiques en cognition sociale, thèse de doctorat, École polytechnique, Paris, 2004.

Chavalarias, David, "Metamimetic games: modeling metadynamics in social cognition », Journal of artificial societies and social simulations, vol. 9, $\mathrm{n}^{\prime \prime} 2,2006$, http://jasss.soc.surtey.ac.uk/9/2/5.html.

Conein, Bernard, Les sens sociaux, Paris, Economica, 2005.

Donald, Merlin, Origins of the modem mind, Cambridge (MA), Harvard University Press, 1991.

Dupuy, Jean-Pierre, «Economic as symptom », dans Transforming econonics. Perspectives on the crifical realist project, Londres et New York, Routledge, 2004, p. 227-251.

Dupuy, Jean-Pierre et Paul Dumouchel (dir.), L'auto-organisation, de la pbysique au politique, Paris, Seuil, 1983.

Frank, Joshua, «Natural selection, rational economic behavior, and altemative outcomes of the evolutionary process "), Journal of socio-economics, vol. 32, n" 6, 2003, p. 601-622.

Girard, René, Mensonge romantique et vérité romanesque, Paris, Grasset, 1961.

Hedstrom, Peter, «Rational Imitation », dans Peter Hedstrom et Richard Swedberg (dir.), Social mechanism. An analytical approach to social theory, Cambridge, Cambridge University Press 1998.

Henrich, Joe et Richard Boyd, "The cvolution of conformist transmission and the emergence of between-group differences ", Evolution and buman behavior, vol. 19, n" 4, 1998, p. 215-241.

Hofstadter, Douglas, Gödel, Escher, Bach: An eternal golden braid, s. 1., New York (NY), Basic Books, 1999.

Hutchins, Edwin, Cognition in the wild, Cambridge (MA), MIT Press, 1995.

Le Moigne, Jcan-I ouis, « Représenter et raisonner les comportements socio-économiques », dans Christine Roland-Iévy et Philippe Adair (dir.), Psychologie économique, théorie et applications, Paris, Economica, 1988, p. 317-337.

Lestel, Dominique, Les origines animales de la culture, Paris, Flammarion, 2001.

Meltzoff, Andrew et Wolfgang Prinz (dir.), The imitative mind: Development, evolution, and brain bases, Cambridge, Cambridge University Press, 2002.

Nadel, Jacqueline et Jean Decety (dir.), Imiter pour decounir l'bumain. Psychologie, neurologie, robotique et philosophie de l'esprit. Psychologie et science de la pensée, Paris, PUF, 2002.

Orléan, André, "Les interactions mimétiques », dans Jacques Lesourne, André Orléan et Bernard Walliser (dir.), Legons de microéconomie évolutionniste, Paris, Odile Jacob, 2002, p. 119-157.

Rabin, Matthew, «A perspective on psychology and economics», European economic review, vol. 46, 2002, p. 657-685.

Selten, Reinhard et Jose Apesteguia, « Experimentally observed imitation and cooperation in price competition on the circle ", Games and economic behavior, vol. 51, n" 1, 2005, p. $171-192$. 
Selten, Reinhard ct Axel Ostmann, «Imitation Equilibrium», Homo oeconomicus, vol. 18, 2001, p. 111-149.

Tarde, Gabriel, I es lois de limitation, les classiques des Sciences Sociales, Université du Québec à Chicoutimi, Jean-Marie Tremblay, [1890] 2002.

Tarde, Gabriel, Les lois sociales, esquisse d'une sociologie, Les classiques des Sciences Sociales, Université du Québec à Chicoutimi, Jean-Marie Tremblay, [1898, Paris, Alcan] 2002.

Tomasello, Michael, The cultural origins of buman cognition, Cambridge (MA), Harvard University Press, 1999.

Varela, Francisco, «L'auto-organisation : de l'apparence au mécanisme », dans Jean-Pierre Dupuy et Paul Dumouchel (dir.), L 'auto organisation, de la physique au politique, Paris, Seuil, 1983, p. 147-164. 